\title{
Characterizing liver sinusoidal endothelial cell fenestrae on soft substrates upon AFM imaging and deep learning
}

\author{
Peiwen $\mathrm{Li}^{\mathrm{a}}{ }^{\mathrm{b}}$, Jin Zhou ${ }^{\mathrm{b}}$, Wang $\mathrm{Li}^{\mathrm{b}, \mathrm{c}}$, Huan $\mathrm{Wu}^{\mathrm{b}, \mathrm{d}}$, Jinrong $\mathrm{Hu}^{\mathrm{b}}$, Qihan Ding ${ }^{\mathrm{b}, \mathrm{c}}$, Shouqin Lü $^{\mathrm{b}, \mathrm{c}}$, \\ Jun Pan ${ }^{\mathrm{d}}$, Chunyu Zhang ${ }^{\mathrm{a}, *}$, Ning Li ${ }^{\mathrm{b}, \mathrm{c}, *}$, Mian Long ${ }^{\mathrm{b}, \mathrm{c}, *}$ \\ ${ }^{a}$ School of Life Science, Beijing Institute of Technology, Beijing 10081, China \\ ${ }^{\mathrm{b}}$ Center for Biomechanics and Bioengineering, Key Laboratory of Microgravity (National Microgravity Laboratory), and Beijing Key Laboratory of Engineered Construction \\ and Mechanobiology, Institute of Mechanics, Chinese Academy of Sciences, Beijing 100190, China \\ ${ }^{\mathrm{c}}$ School of Engineering Sciences, University of Chinese Academy of Sciences, Beijing 100049, China \\ ${ }^{\mathrm{d}}$ Key Laboratory of Biorheological Science and Technology (Chongqing University), Ministry of Education, Chongqing 400044, China
}

\section{A R T I C L E I N F O}

\section{Keywords:}

Liver sinusoidal endothelial cells

Fenestrae

Atomic force microscopy

Imaging recognition

Fully convolutional networks

Substrate stiffness

\begin{abstract}
A B S T R A C T
Background: Liver sinusoidal endothelial cells (LSECs) display unique fenestrated morphology. Alterations in the size and number of fenestrae play a crucial role in the progression of various liver diseases. While their features have been visualized using atomic force microscopy (AFM), the in situ imaging methods and off-line analyses are further required for fenestra quantification.

Methods: Primary mouse LSECs were cultured on a collagen-I-coated culture dish, or a polydimethylsiloxane (PDMS) or polyacrylamide (PA) hydrogel substrate. An AFM contact mode was applied to visualize fenestrae on individual fixed LSECs. Collected images were analyzed using an in-house developed image recognition program based on fully convolutional networks (FCN).

Results: Key scanning parameters were first optimized for visualizing the fenestrae on LSECs on culture dish, which was also applicable for the LSECs cultured on various hydrogels. The intermediate-magnification morphology images of LSECs were used for developing the FCN-based, fenestra recognition program. This program enabled us to recognize the vast majority of fenestrae from AFM images after twice trainings at a typical accuracy of $81.6 \%$ on soft substrate and also quantify the statistics of porosity, number of fenestrae and distribution of fenestra diameter.

Conclusions: Combining AFM imaging with FCN training is able to quantify the morphological distributions of LSEC fenestrae on various substrates.

Significance: AFM images acquired and analyzed here provided the global information of surface ultramicroscopic structures over an entire cell, which is fundamental in understanding their regulatory mechanisms and pathophysiological relevance in fenestra-like evolution of individual cells on stiffness-varied substrates.
\end{abstract}

\section{Introduction}

Liver sinusoidal endothelial cells (LSECs) are highly specialized endothelial cells which constitute the hepatic sinusoidal wall and act as the permeable barrier between blood stream and surrounding hepatocytes $[1,2]$. In healthy liver, LSECs display unique morphology characterized by the presence of non-diaphragmed transcellular pores, or so-called fenestrae, and the lack of basement membrane [3]. These structural and phenotypic features maintain proper liver functions and homeostasis, promote efficient trafficking of lipoproteins, and regulate liver regeneration and immune tolerance $[1,2,4,5]$. The sizes of LSEC fenestrae range from approximately 50 to $200 \mathrm{~nm}$ in diameter and the majority of them are grouped into several-tens ultrastructures termed as sieve plates [6,7]. Alterations in the size and number of fenestrae play a

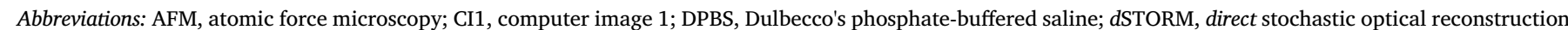

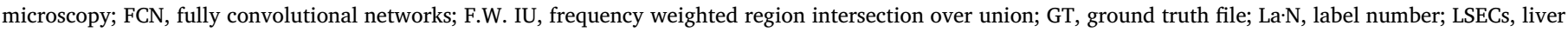

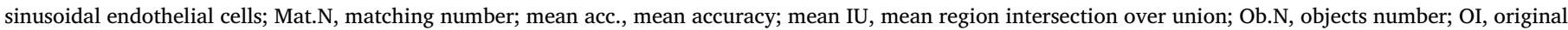

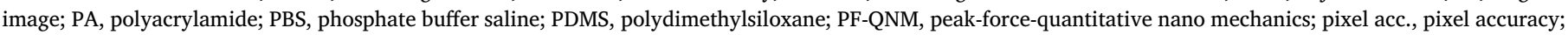
SEM, scanning electron microscopy; STED, stimulated emission depletion; 3D-SIM, three-dimensional structured illumination microscopy.

* Corresponding authors.

E-mail addresses: zzccyy1977@bit.edu.cn (C. Zhang), lining_1@imech.ac.cn (N. Li), mlong@imech.ac.cn (M. Long). 
crucial role in the progression of various liver diseases, including diabetes mellitus, acute or chronic liver injury induced by hormones, alcohol, or drugs, as well as liver fibrosis and cirrhosis [3,8]. Thus, the visualization and quantification of LSEC fenestrae are of great interest to understand the pathophysiological processes and explore new therapeutic strategies.

The size of fenestrae is usually smaller than the optical resolution limit and no specific surface markers for fenestrae have been found to date [1,9]. As a result, their observations are largely relied upon scanning electron microscopy (SEM) in the literature [10]. Applying SEM requires multi-step sample preparation, including fixation, dehydration, drying, and gold sputtering coating [11], leading to the potential cell contraction and physicochemical damage to the cell surface and then resulting in additional pores and measurement errors [3]. Recently, several super-resolution fluorescence techniques, such as three-dimensional structured illumination microscopy (3D-SIM), direct stochastic optical reconstruction microscopy (dSTORM), and stimulated emission depletion (STED) microscopy, were used to visualize the presence of fenestrae and their co-localization with actin filaments [12-15]. These techniques usually require LSECs seeded on glass coverslips with specific thickness or refractive index, which may constrict the selection of seeding substrates and compromise LSEC phenotype since a typical stiff substrate $(36 \mathrm{kPa})$ can lead to loss of fenestrae $[16,17]$. Atomic force microscopy (AFM) is another label-free technique recently used to visualize fenestrae in fixed or live LSECs $[6,8,18]$. Time-lapse imaging of live LSECs by AFM is able to reveal the lifespan, formation and disappearance of LSEC fenestrae [19]. One obvious advantage of this approach is that AFM tips scan directly the upper surface of the samples, which is irrelevant to the seeding substrates. However, the methodology of AFM imaging and off-line analysis of LSEC fenestrae is still preliminary and needs to be further optimized.

To evaluate the alterations and regulating factors of fenestra evolution, the number and diameter distributions of fenestrae have to be quantitatively determined from the acquired images. Previous studies usually analyzed AFM images with ImageJ software manually or semiautomatically by threshold segmentation $[6,15]$. The former is laborconsuming and the latter requires high contrast between fenestrae and the surrounding membrane. Thus, it is critical to establish a set of intelligent, efficient and self-adaptive methods for fenestra recognition and quantification. Deep learning is a good candidate in this regard, since it is originated from machine learning and applies a set of simple algorithms to implement the high-level data abstractions by tagging the raw data from human experiences. Trained deep learning neural networks can replace manual labors and have been applied in cell recognition [20]. For example, a fully convolutional network (FCN) is one typical network that has less complexity in preprocessing and postprocessing but successfully used to predict the semantic segmentation of size-irrelevant images and improve the accuracy and efficiency through end-to-end image block training [21,22]. Therefore, developing FCN-based algorithms could be helpful in accurate and fast recognition of LESC fenestrae.

Here we attempted to optimize AFM scanning parameters for visualizing the fenestrae on LSECs seeded directly on culture dish or on hydrogels pre-immobilized on the dish. Moreover, an image recognition program based on FCN was developed to estimate the porosity, number of fenestrae, and distribution of fenestra diameter.

\section{Materials and methods}

\subsection{Cell isolation and culture}

\subsubsection{Cell isolation}

Primary murine LSECs were isolated from 6 to 8 weeks old male C57BL/6 mice (Vital River Laboratories, Beijing, China) after approval by the Institutional Animal and Medicine Ethical Committee at the Institute of Mechanics, Chinese Academy of Sciences, as previously described [23,24]. Briefly, the mouse liver was perfused in situ from the portal vein with a $\mathrm{Ca}^{2+}$-free Gey's balanced salt solution at a rate of $5 \mathrm{ml} \mathrm{min}^{-1}$ for $5 \mathrm{~min}$ and then switched to a $0.05 \%$ collagenase IV solution (Sigma-Aldrich, St. Louis, MO, USA) at a rate of $5 \mathrm{ml} \mathrm{min}^{-1}$ for an additional $5 \mathrm{~min}$. The liver was then minced, and harvested in high glucose DMEM medium (Hyclone, Logan, UT, USA). The homogenate was filtered through a cell strainer $(200 \mu \mathrm{m}$ in diameter) to remove undigested tissue sediments and centrifuged at $54 \times g$ at $4{ }^{\circ} \mathrm{C}$ for $3 \mathrm{~min}$ twice. The collected supernatant was centrifuged at $500 \times g$ for $8 \mathrm{~min}$, and the sediments were resuspended with $3 \mathrm{ml}$ of a $24 \%$ Optiprep solution (Axis-Shield, Dundee, Scotland) in high glucose DMEM medium, followed by $3 \mathrm{ml}$ of $17.6 \%, 11.7 \%$ Optiprep solutions and DMEM gently. After density gradient equilibrium centrifugation at $1400 \times g$ for $18 \mathrm{~min}$ at $20{ }^{\circ} \mathrm{C}$, non-parenchymal cells containing LSECs were collected from the layer between $11.7 \%$ and $17.6 \%$ Optiprep and washed with Dulbecco's phosphate-buffered saline (DPBS, Hyclone). Collected cells were then incubated with $10 \mu \mathrm{g} / \mathrm{ml}$ FITC-conjugated rat-antimouse CD146 and PE-conjugated recombinant human-anti-mouse F4/ 80 monoclonal antibodies (mAbs) (Miltenyi Biotec, Bergisch Gladbach, Germany) at $4{ }^{\circ} \mathrm{C}$ in the dark for $15 \mathrm{~min}$. Washed cells were used for flow cytometry sorting by FACS Aria III (BD Biosciences, Franklin Lakes, NJ, USA), where LSECs were isolated by $\mathrm{CD} 146^{+} \mathrm{F} 4 / 80^{-}$gating.

\subsubsection{Cell culture}

After the isolation, LSECs were seeded on rat tail collagen type Icoated (Shengyou Biotechnology, Hangzhou, China) culture dish or polydimethylsiloxane (PDMS) or polyacrylamide (PA) hydrogel substrates pre-placed on the dish. Cells were cultured in Endothelial Cell Medium (Sciencell Research Laboratories, Carlsbad, CA, USA) at $37{ }^{\circ} \mathrm{C}$ with $5 \% \mathrm{CO}_{2}$ for 6 to $24 \mathrm{~h}$, then fixed with $1.0 \%$ glutaraldehyde (Aladdin, Shanghai, China) for $10 \mathrm{~min}$ and stored in DPBS (Hyclone) before use. In some cases, live cells were treated with $5 \mu \mathrm{M}$ cytochalasin D (Tocris Bioscience, Bristol, UK) before AFM imaging.

\subsection{Preparation of PDMS and PA hydrogel}

\subsubsection{PDMS substrates}

PDMS (Dow Corning, Midland, MI, USA) with a nominal elastic modulus of 1.86 MPa was mixed up by a silicone elastomer base and a curing agent completely in a weight ratio of 10: 1 (base: curing agent) [25]. The PDMS mixture was poured onto a piece of glass, degassed with a vacuum desiccator (Yilibotong Company, Beijing, China), and then fully cured in an oven at $85{ }^{\circ} \mathrm{C}$ for $1 \mathrm{~h}$.

\subsubsection{PA hydrogel substrates}

PA hydrogel with a nominal elastic modulus of $1050 \mathrm{~Pa}$ was prepared as previously described [26-30]. Briefly, $0.075 \mathrm{ml}$ of $40 \%$ acrylamide (Sigma-Aldrich), $0.05 \mathrm{ml}$ of bis-acrylamide (Sigma-Aldrich), $0.1 \mathrm{ml}$ of $10 \times$ PBS (Solarbio, Beijing, China), $0.05 \mathrm{ml}$ of $1 \%$ tetramethylethylenediamine (Sigma-Aldrich), $0.05 \mathrm{ml}$ of $1 \%$ potassium persulfate (Sigma-Aldrich), and $0.675 \mathrm{ml}$ of ultra-pure water were mixed to form a solution with a final concentration of $3 \%$ acrylamide and $0.1 \%$ bis-acrylamide. $100 \mu \mathrm{l}$ mixture was added onto the hydrophilic-treated glass-bottom dish, covered with an $18 \mathrm{~mm}$ diameter circular coverslip to allow the hydrogel to be polymerized for $10 \mathrm{~min}$, and rinsed with ultra-pure water overnight. The PA hydrogel surfaces were then conjugated to $\mathrm{N}$-succinimidyl acrylamidohexanoic acid (VWR, Radnor, PA, USA) and activated for $15 \mathrm{~min}$ by $306 \mathrm{~nm}$ ultraviolet exposure and Irgacure 2959 (Sigma-Aldrich) catalyst immediately before coating with $100 \mu \mathrm{g} / \mathrm{ml}$ rat tail collagen I (Shengyou Biotechnology, Hangzhou, China) overnight.

\subsection{AFM measurements}

AFM imaging was conducted at room temperature in PBS using a Bioscope Catalyst (Bruker Corporation, Billerica, MA, USA). Two 
different AFM working modes, contact mode and PeakForceQuantitative Nano Mechanics mode (PF-QNM) mode [31], were applied in the measurements. All AFM probes with reflective gold-coated triangular cantilevers and pyramidal tips were purchased from Bruker Corp, in which MLCT-C and MSNL-10-C cantilevers were used in contact mode while MLCT-E and Scansyst Fluid cantilevers were used in PF-QNM mode. The external dimensions and spring constant of the cantilevers were summarized in Table S1. NanoScope analysis software (Bruker Corporation, v1.80) was used for generating the images of cellular morphology (deflection error) and height.

\subsection{Development of FCN for fenestra tracing}

\subsubsection{Manual annotation of fenestrae}

The color scale of LSEC morphology images collected above were first adjusted with NanoScope analysis software to make the fenestrae darker than surrounding membrane and easier to be identified by human eyes. The modified images were then input into an in-house developed Label software upon MATLAB (v8.3, MathWorks, Natick, MA, USA). All fenestrae with typical characteristics and clear contours in every image were recognized by experience and labeled manually along their outer edges. There should be no overlap between the neighboring labels. The images were finally converted into binary images by setting the labeled fenestrae to white, and the background to black. A ground truth (GT) file of each picture for these fenestrae was then generated from the Label software.

\subsubsection{FCN training}

Fenestra identification in localized LSEC images can be formulated as a semantic segmentation problem and thus a FCN framework adopted from literature [21] was employed to recognize the fenestrae. Here FCN was trained on four NVIDIA GTX1080Ti GPU with 11 GB GDDR5 RAM using cuda 8.0 and cuDNN 5 with caffe framework [32] under Linux system (Ubuntu16.04, Canonical, London, UK). In the firstrun training, total 107 localized images and their corresponding GT files 1 (GT1) were employed, of which 93 images and their label files were augmented six times by horizontal reflection and clockwise rotation for reducing the overfitting and then divided into both the training and validating sets with a ratio of 7 to 2 while the remaining 14 images served as the testing set. The training was conducted for 10 epoches, in which each epoch included 5000 iterations and the FCN was validated every epoch. Four metrics from common semantic segmentation and scene parsing evaluations were applied as used in the literature [21], i.e., pixel accuracy (shorted as, pixel acc.), mean accuracy (mean acc.), mean region intersection over union (mean $I U$ ) and frequency weighted IU (F.W. IU). Let $n_{i j}$ be the number of pixels of class $i$ predicted to belong to class $j$ in total $n_{c l}$ different classes and $t_{i}=\Sigma_{j} n_{j i}$ be the total number of pixels of class $i$. The above four matrices were thus calculated as pixel acc. $=\Sigma_{i} n_{i i} / \Sigma_{i} t_{i}$, mean acc. $=\left(1 / n_{c l}\right) \Sigma_{i} n_{i i} / t_{i}$, mean IU $=\left(1 / n_{c l}\right) \Sigma_{i} n_{i i} /\left(t_{i}+\Sigma_{j i} n_{j i}-n_{i i}\right)$, and F.W. IU: $\left(\Sigma_{k} t_{k}\right)^{-1} \Sigma_{i} t_{i} n_{i i} /$ $\left(t_{i}+\Sigma_{j} n_{j i}-n_{i i}\right)$. After 10 epoches, the first-trained FCN model (FCN1) was obtained.

\subsubsection{FCN retraining}

The FCN1 model was then retrained since the mean $I U$ estimated was lower than the levels reported in the literature [21] and the resulted fenestra size, shape and location were different from the manual identification in the GT1. Here 82 images with clear structural features of fenestrae were screened out of the first-run training, and pooled together with 32 newly-added images. Specifically, the fenestra tracing of 82 images used to train FCN1 was profiled by FCN1 to obtain 82 images with labeled fenestrae, which was named as computer image 1 (CI1). These fenestrae in CI1 were compared with their original images and the GT file of CI1 was modified when fenestra shape or size could not match or the fenestrae were overlapped with other fenestrae, which was named as GT2 file. 82 original images with GT2 and 32 new images with their GT file were again augmented six times and divided into both training and validating sets with a ratio of 7 to 2 while the testing set was as same as the one in the first-run training. The second-run training was conducted also in terms of epoches, and the FCN2 was obtained after 20 epoches.

\subsubsection{Evaluation metrics of FCN testing set}

To compare the consistency of LSEC fenestra recognition between FCN models and manual identification, 14 images in the testing set were employed to evaluate the similarity of fenestra size, shape, location, and number accuracy by Dice similarity coefficient (Dice) [33] and $F_{1}$ score [34] defined as, respectively,

$$
\begin{aligned}
& \operatorname{Dice}(X, Y)=\frac{2(X \cap Y)}{(X+Y)} \text {,and } \\
& F_{1} \text { score }=\frac{2 \times T P}{2 \times T P+F P+F N}
\end{aligned}
$$

where $X$ and $Y$ are the readouts of respective FCN model and manual identification, TP and FP are the numbers of true positive and false positive values, respectively, and $F N$ is the number of false negatives based on overlapped pixels in FCN model and manual identification. In addition, three common biological indicators of the porosity, number of fenestrae, and distribution of fenestra diameter [35] were used to demonstrate the fenestra recognition. Here porosity is the ratio of the fenestra area to the cell area. The fenestra morphology identified by FCN model was approximately circular and therefore the diameter of equivalent area was determined to estimate fenestra diameter distribution.

STAR $\star$ Methods.

\begin{tabular}{|c|c|c|}
\hline Reagent or resource & Source & Identifier \\
\hline \multicolumn{3}{|l|}{ Antibodies } \\
\hline CD146 & Miltenyi Biotec & $130-102-230$ \\
\hline $\mathrm{F} 4 / 80$ & Miltenyi Biotec & $130-116-499$ \\
\hline \multicolumn{3}{|c|}{ Chemicals, Peptides, and Recombinant Proteins } \\
\hline Optiprep & Axis-Shield & 1114542 \\
\hline Glutaraldehyde & Aladdin & G105906 \\
\hline Polydimethylsiloxane & Dow Corning & DC184 \\
\hline Cytochalasin D & Tocris & $1233 / 5$ \\
\hline & Bioscience & \\
\hline Acrylamide & Sigma & A3553 \\
\hline Bis-acrylamide & Sigma & M1533 \\
\hline Tetramethylethylenediamine & Sigma & T9281 \\
\hline Potassium persulfate & Sigma & 216224 \\
\hline Rat tail tendon collagen type I & Shengyou & 200110-10 \\
\hline & Biotechnology & \\
\hline $\begin{array}{l}\text { N-succinimidyl Acrylamidoh- } \\
\text { exanoic acid }\end{array}$ & VWR & N/A \\
\hline Irgacure-2959 & Sigma & 410896 \\
\hline \multicolumn{3}{|c|}{ Experimental Models: Organisms/Strains } \\
\hline C57BL/6 N & $\begin{array}{l}\text { Vital River } \\
\text { Laboratories }\end{array}$ & 213 \\
\hline \multicolumn{3}{|l|}{ Software and Algorithms } \\
\hline Matlab & MathWorks & $\begin{array}{l}\text { https://www.mathworks.com/ } \\
\text { products/matlab.html?s_tid = hp_ } \\
\text { products_matlab }\end{array}$ \\
\hline Caffe Framework & $\begin{array}{l}\text { Berkeley } \\
\text { Vision and } \\
\text { Learning } \\
\text { Center (BVLC) }\end{array}$ & http://caffe.berkeleyvision.org/ \\
\hline $\begin{array}{l}\text { Ubuntu Linux } \\
\text { NanoScope Analysis }\end{array}$ & $\begin{array}{l}\text { Canonical Ltd } \\
\text { Bruker }\end{array}$ & $\begin{array}{l}\text { https://ubuntu.com/ } \\
\text { https://www.brukersupport.com/ }\end{array}$ \\
\hline
\end{tabular}

Key Resources Table.

\section{Results and discussions}

\subsection{Visualizing the fenestrae of LSECS on culture dish}

We first visualized the fenestrae of LSECs on culture dish using AFM 

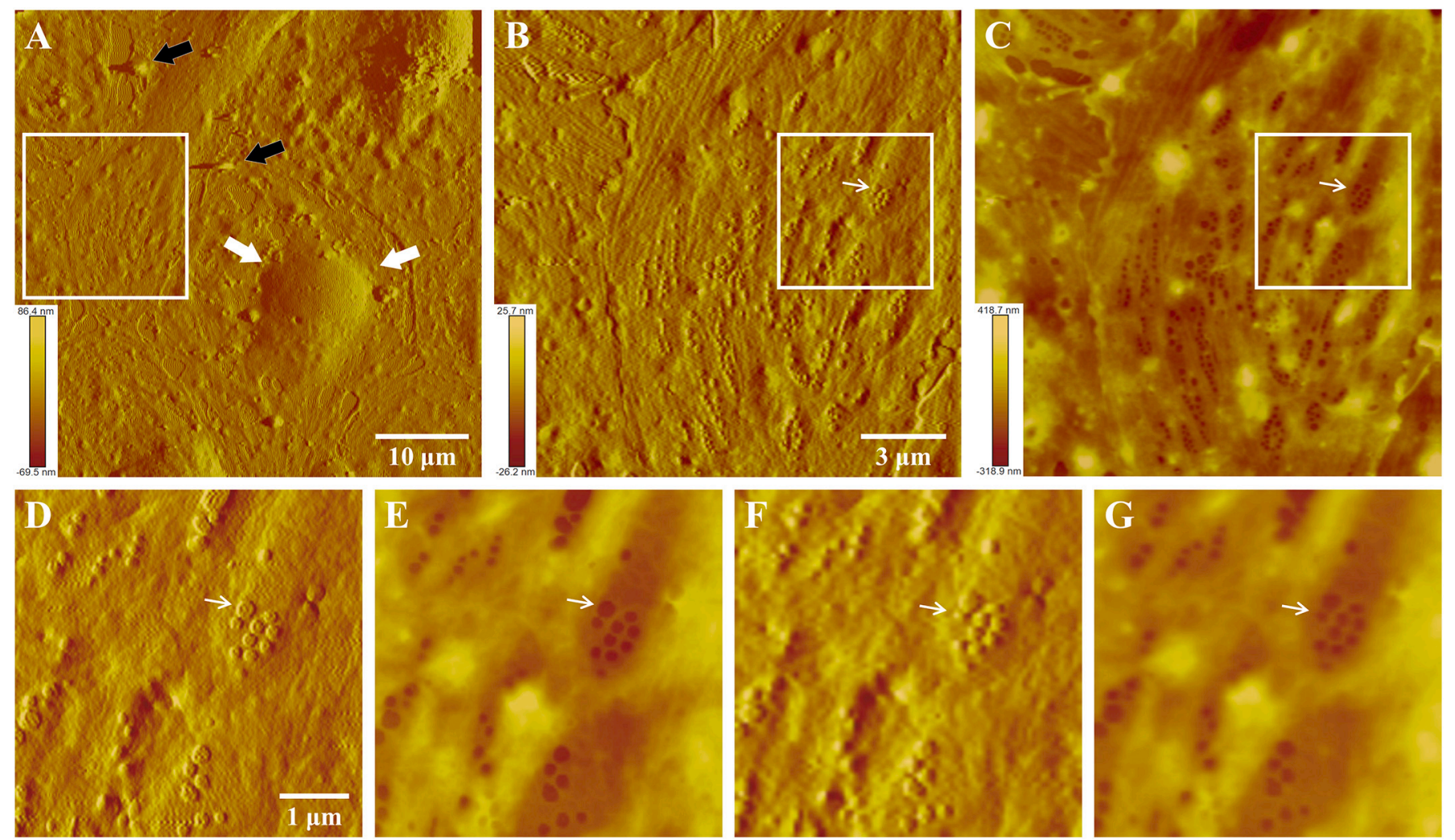

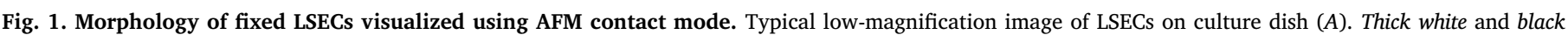

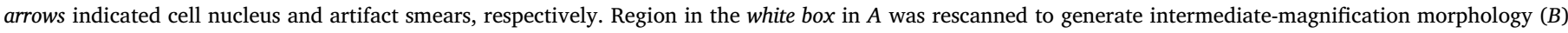

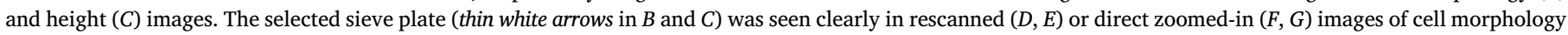

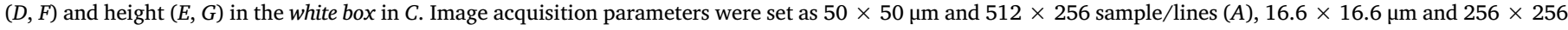
sample/lines $(B, C, F, G)$, and $5 \times 5 \mu \mathrm{m}$ and $256 \times 256$ sample/lines $(D, E)$. MLCT-C cantilever was applied and loading force was $0.89 \mathrm{nN}$ for all images.

assay. Here the same cell was scanned for 1-10 times to optimize scanning parameters (i.e., image size and resolution (sample/lines) determine scanning speed). Notably, the side effects of multiple scanning such as tip wear or sample damage could be ignored since no difference in fixed LSECs was observed up to the 10th scanning (Fig. S1). Collected AFM images provided the elaborative information of surface morphology, cell height, as well as LSEC fenestrae and sieve plates (Fig. 1). Images at low magnification $(50 \times 50 \mu \mathrm{m})$ enabled the visualization of LSECs in contact mode at a speed of $8 \mathrm{~min} /$ picture with clear contrast between the cell membrane or nucleus and the background (Fig. 1A). Intermediate-magnification $(16.6 \times 16.6 \mu \mathrm{m})$ images at cell periphery were acquired efficiently at $256 \times 256$ sample/lines to obtain a single image within $4 \mathrm{~min}$ (Fig. 1B, C). Rescanning smaller observation window at higher resolution (Fig. 1D, E) provided similar information for visualizing fenestrae as zoom-in (Fig. 1F, G). Thus, the intermediate-magnification images $(16.6 \times 16.6 \mu \mathrm{m}, 256 \times 256$ sample/lines) were selected in the following fenestra recognition to balance the accuracy and the efficiency of data acquisition. Previous AFM measurements usually focused on the clarity of fenestra images and only covered several sieve plates in a single image $(<5 \times 5 \mu \mathrm{m})$ $[6,8,18]$. While these pioneering works are able to demonstrate the existence of LSEC fenestrae, it is still hard to elaborate their biological relevance since the sieve plates on LSECs are unevenly distributed. Enlarging the image size is able to offer the global information over an entire cell for fenestra distribution in various conditions.

In addition to the image size and resolution, loading force and tip apex radius were also assumed to affect the quality of AFM images for LSECs $[6,8,18]$. The MLCT-C cantilever was usually chosen, due to its low spring constant, to enable the visualization of the fenestrae in contact mode [8]. When the loading force was set to $0.89 \mathrm{nN}$, a clear fenestra structure was obtained (Fig. 2A, E). Increasing loading force enlarged the diameter of fenestrae observed (Fig. 2A-H) but reduced the cell height gradually (Fig. 2I). Setting the highest loading force of $8.88 \mathrm{nN}$ made it impossible to obtain a clear image (Fig. 2D, H). We also tested the applicability of another AFM cantilever, MSNL-10-C cantilever, with the same spring constant but a sharper tip. Consistently, applying MSNL-10-C cantilever exhibited the enlarged fenestra structure with higher loading force (Fig. S2) but was not able to map the fenestrae at $4.07 \mathrm{nN}$. This sharper tip increased the pressure applied to the cell surface, resulting in the potential disruption of fenestrae at same loading force. When the loading force was less than $1 \mathrm{nN}$, the acquired high-magnification images showed that MSNL-10-C cantilever was still able to map the clearer (arrows in Fig. 3) and larger (arrowheads in Fig. 3) fenestrae than MLCT-C cantilever. These enlarged fenestrae were similar to those obtained at high loading force (cf. Fig. 2), suggesting that sharper tip and higher force could independently cause the image distortion. Moreover, the colored zone in the cross-section can be used to clarify the fenestra boundaries (Fig. 3C, F), where a smaller angle stood for the clearer fenestrae image for MSNL-10-C cantilever. Collectively, although the sharper tip contributed to higher resolution, the following fenestra imaging was performed using regular MLCT-C cantilever at loading force of $0.89 \mathrm{nN}$ to prevent potential fenestra structure damage induced by higher pressure with sharper tips.

\subsection{Visualizing the fenestrae of LSECs on biologically-relevant substrates}

The above optimization is not only critical to obtain high-quality fenestra images on culture dish, but it also serves as a prerequisite to implement the fenestra imaging on biologically-relevant substrates. Increasing evidence supports that mechanical stimuli regulate the morphology, proliferation, and differentiation of cells placed on PDMS or PA hydrogel substrates with pathophysiologically-mimicking 


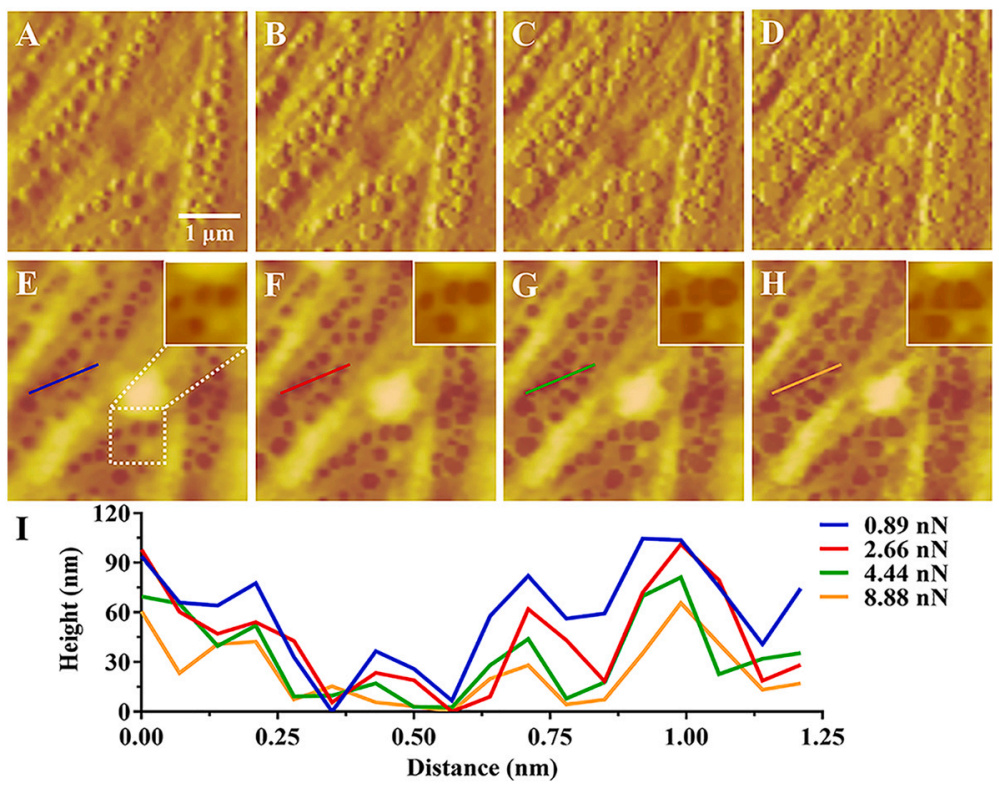

Fig. 2. Morphology of fixed LSECs visualized at various loading forces in contact mode. Loading force was set to $0.89(A, E), 2.66(B$, $F), 4.44(C, G)$ and $8.88 \mathrm{nN}(D, H)$, respectively. Fenestrae were visible both in morphology $(A-D)$ and height images $(E-G)$. The partially enlarged images were presented in the inserted boxes. Height profiles at various loading forces $(I)$ were plotted along the lines indicated in $E-H$. Image acquisition parameters were set as $16.6 \times 16.6 \mu \mathrm{m}$ and $256 \times 256$ sample/lines for all images. Only partial images were enlarged from original pictures and displayed for clarity. MLCT-C cantilever was applied. stiffnesses $[29,36]$. In the liver, the consistent correlation between enhancement of hepatic tissue stiffness and development of liver fibrosis and cirrhosis implies that tissue stiffness might play a key role in hepatic cell behaviors [16] and, thus, the fenestra formation. Technically, AFM imaging assay endows the ability to visualize nanoscale structures of LSECs on stiffness-varied substrates. Thus, we also tested the fenestra characteristics of LSECs placed on two stiffnesses. Here both clear morphology and height maps were obtained on PDMS substrate (1.86 MPa) (Fig. 4A, B). However, only clear morphology (Fig. 4C) but blurred height was observed on PA hydrogel (1050 Pa) (Fig. 4D). Here a low integral gain was required when imaging LSECs on PA hydrogel, otherwise the system became unstable presumably due to its low stiffness and high viscosity (data not shown). The gain value in the feedback panel determines the feedback on piezo height via error amendment and the feedback loop keeps the deflection signal constant by adjusting the height of piezo tube. To collect the optimal height map, the feedback gain value needs to be increased so that the tip could track the sample surface with minimal cantilever deflection. Thus, a low gain value could result in a blurred height map but not a morphology map. This speculation was verified in LSEC imaging on culture dishes. Clear height map (Fig. S3A, B) was obtained at high gain value but blurred one observed at low gain value (Fig. S3C, D), regardless of clear morphology images in both cases. Since soft substrates favor the maintenance of LSEC phenotype [17], the application of AFM imaging in
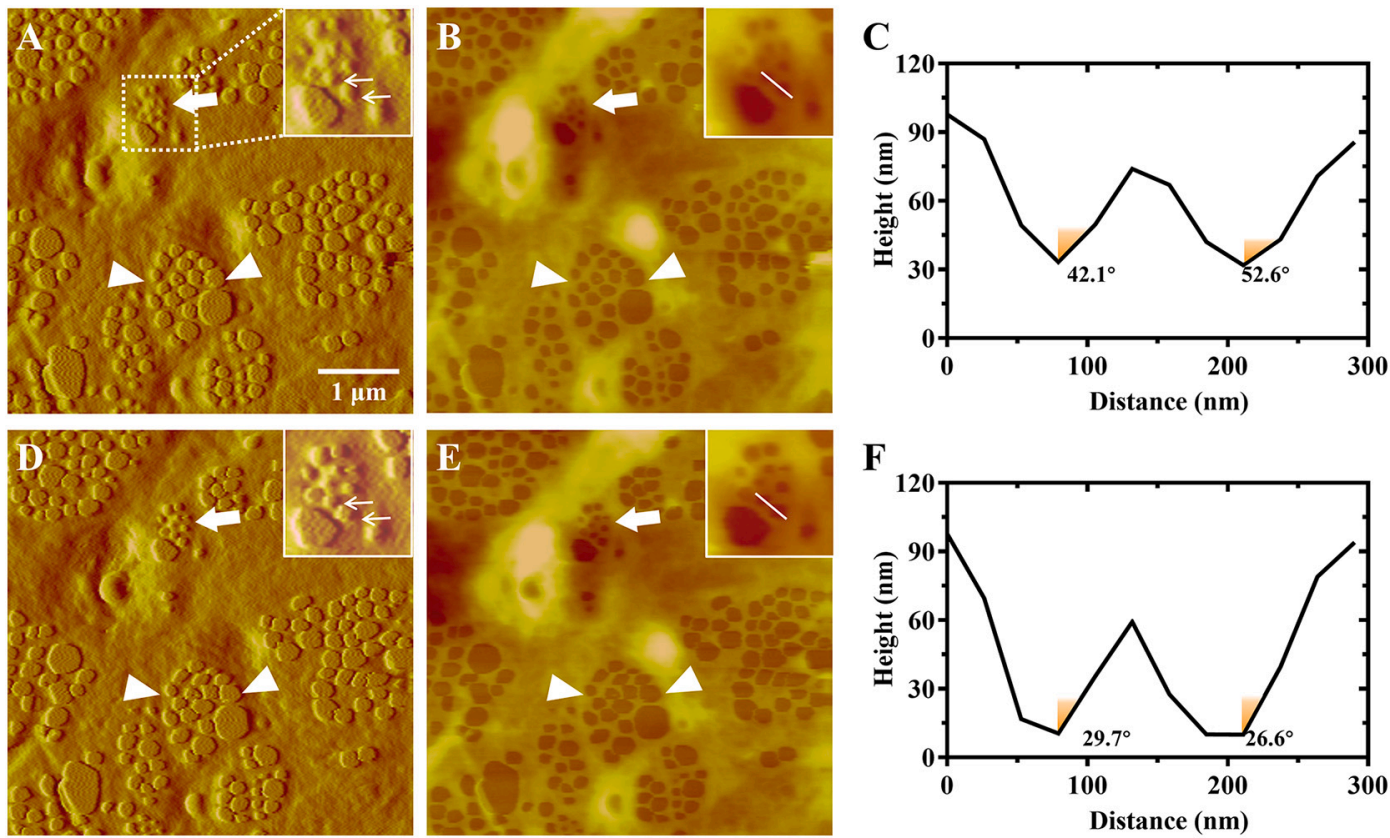

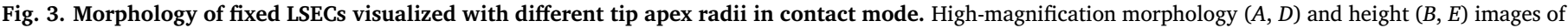

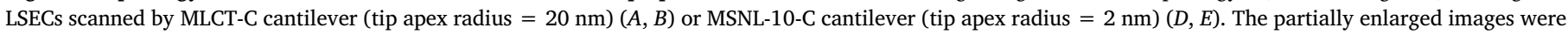

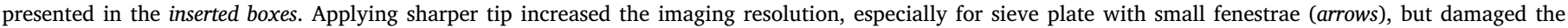

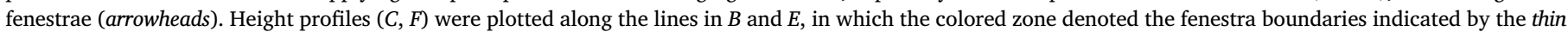

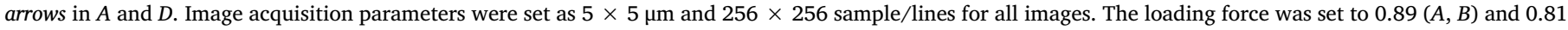
$\mathrm{nN}(D, E)$, respectively. 

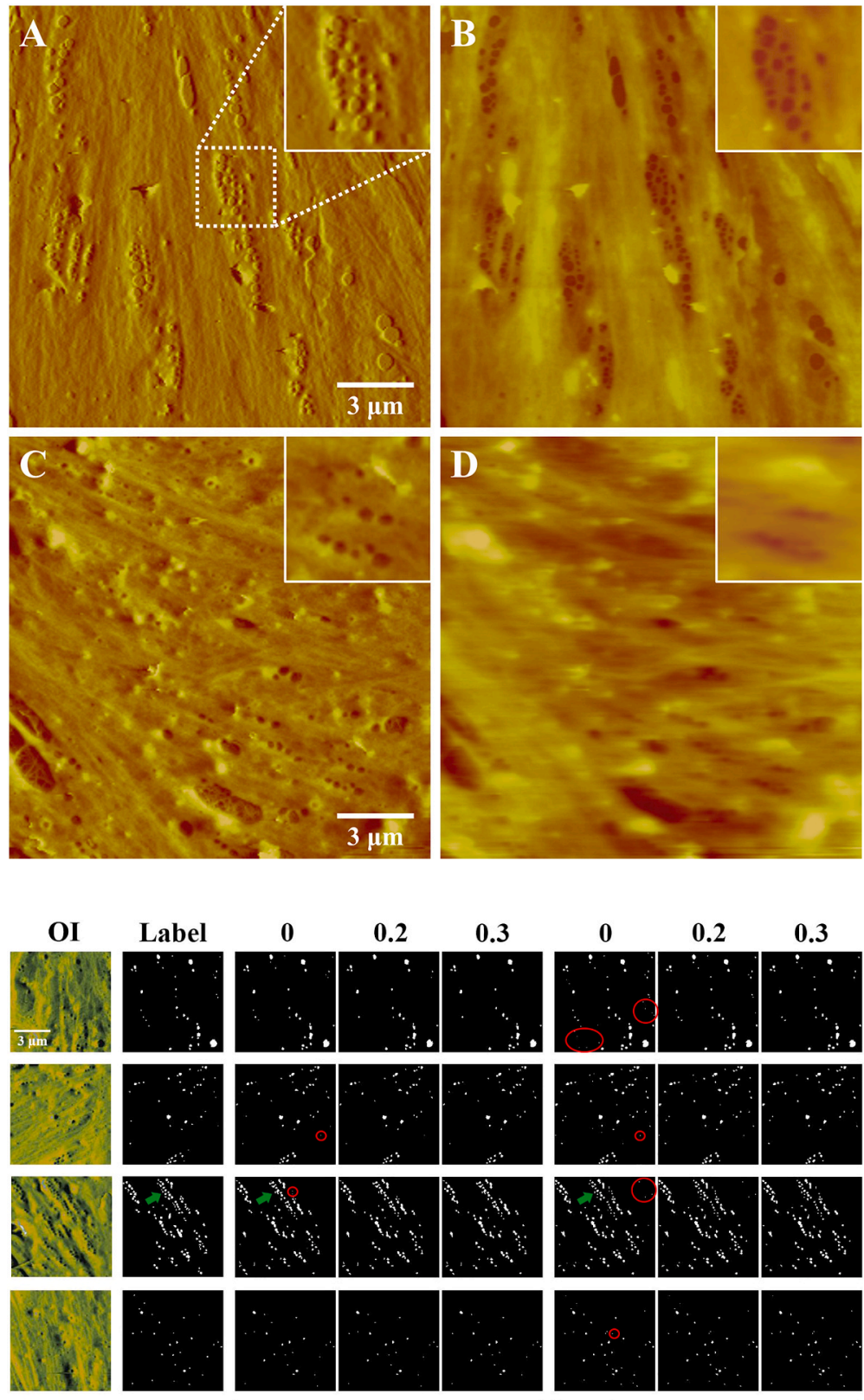

$1^{\text {st }}$ training

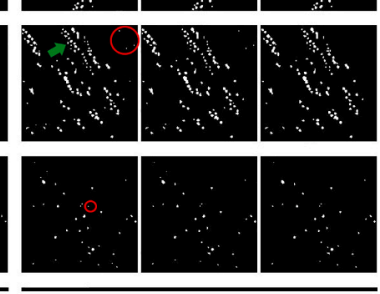

$2^{\text {nd }}$ training
Fig. 4. Morphology of fixed LSECs on various soft substrates visualized using contact mode. Intermediate-magnification morphology $(A, C)$ and height $(B, D)$ maps of LSECs placed on PDMS $(1.86 \mathrm{MPa})(A, B)$ or PA hydrogel $(1050 \mathrm{~Pa})(C, D)$. The partially-enlarged images were presented in the inserted boxes. Image acquisition parameters were set as $16.6 \times 16.6 \mu \mathrm{m}$ and $256 \times 256$ sample/lines. MLCT-C cantilever was applied and loading force was $0.89 \mathrm{nN}$ for all images.

Table 1

Summaries of fenestra recognition by FCN.

\begin{tabular}{|c|c|c|c|c|c|c|c|c|c|}
\hline & pixel acc. & mean acc. & mean IU & F.W.IU & $L a \cdot N^{\mathrm{a}}$ & $O b . N^{\mathrm{b}}$ & Mat. $N^{\mathrm{c}}$ & Dice & $F_{1}$ score \\
\hline FCN1 & 0.977 & 0.660 & 0.616 & 0.959 & 996 & $713(914)^{\mathrm{d}}$ & $641(750)$ & $0.727(0.668)$ & $0.751(0.786)$ \\
\hline FCN2 & 0.980 & 0.688 & 0.658 & 0.963 & 996 & 967 (1236) & $813(884)$ & $0.773(0.739)$ & $0.831(0.785)$ \\
\hline
\end{tabular}

a : Label number of fenestrae in 14 images.

b : Object number by FCN recognition in 14 images.

c : Matching number of fenestrae between manual labeling and FCN in 14 images.

d : Numbers with or without parentheses denoted the objects by setting 0 or 0.2 area threshold.

fenestra identification on soft substrate is physiologically meaningful. Unfortunately, here we failed to obtain clear height map on PA hydrogel, despite of those feedback gain values tested, probably due to flexible deformation of the hydrogel on contact mode. Clear morphology images on soft hydrogel provide sufficient information for fenestra structures but are too difficult to analyze by adjusting intensity thresholds, making it necessary to develop the fenestra recognition program in the next subsection.

On the other hand, force $v s$. distance curves are also applied in highspeed mapping of morphology of fixed and live LSECs $[6,8,18,37]$. The advantage of this imaging mode is to provide both the fenestra morphology and the mechanical features at the same time. PF-QNM mode is 

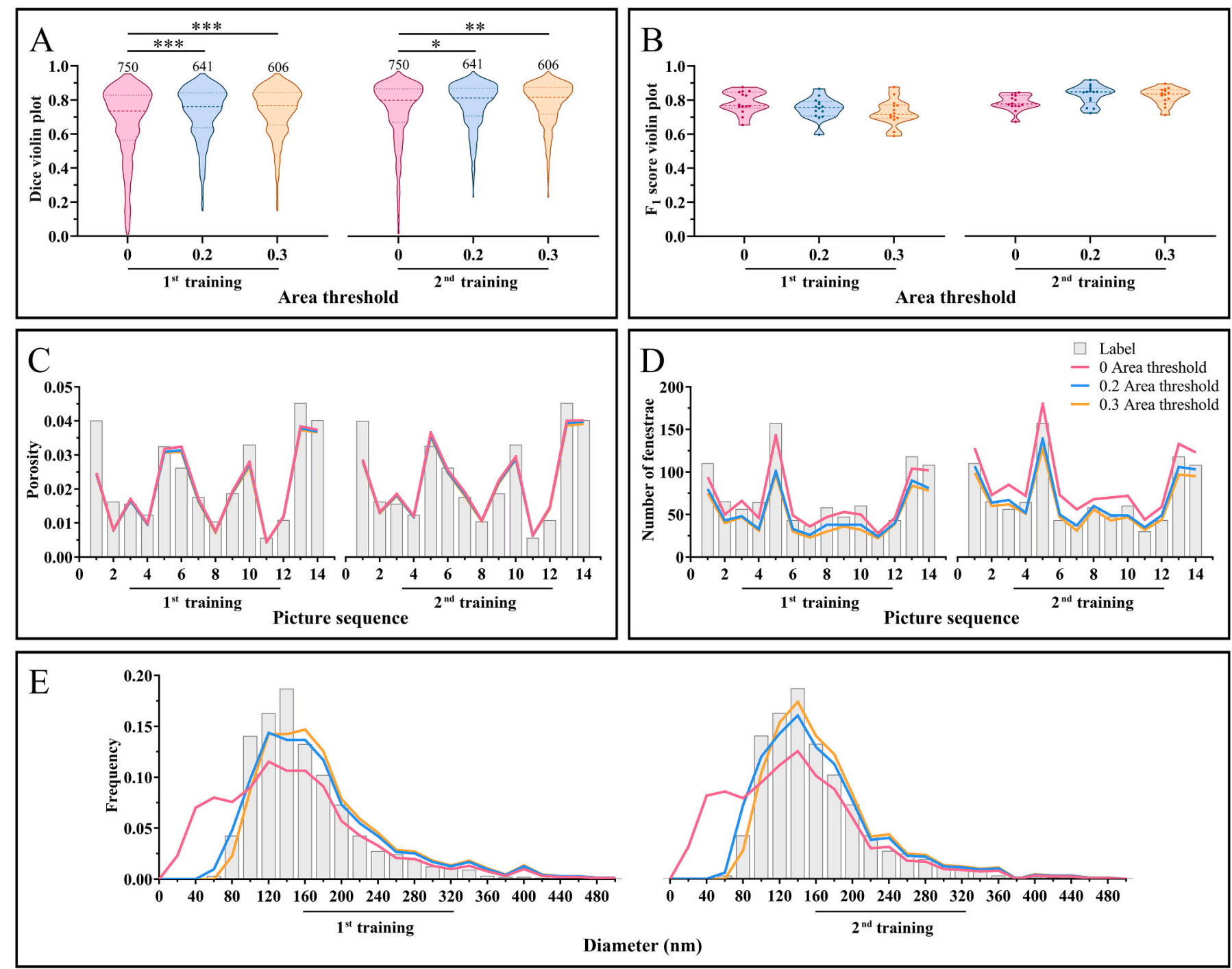

Fig. 6. Recognition readouts of FCN from twice-trained FCN models. $(A, B)$ Violin plots of Dice and $F_{1}$ score. Data were presented as the median values with 25 th and 75th percentiles of sample size inserted $(A)$ and 14 images tested $(B)$. Statistical analysis was conducted by Kruskal-Wallis test followed by Dunn's test. $P<0.05^{*}, 0.01^{* *}$, and $0.001^{* * *}$. $(C, D, E)$ Fluctuation of the estimated porosity $(C)$, number of fenestrae $(D)$, and distribution of fenestra diameter $(E)$ between manual annotation and FCN recognition. Label indicated the fenestra annotated manually. The values of $0,0.2$, and 0.3 indicated the given area thresholds.

a similar mode released by Bruker Corporation, which can be used for mapping the cell height, topography, and elasticity simultaneously $[31,38]$. Evidently, applying PF-QNM mode here enabled to visualize fenestrae of fixed LSECs (Fig. S4) and monitor the time-lapsed fenestra evolution in live LSECs (Fig. S5) on culture dish, mainly attributed to the stiffness difference between the stiff dish bottom inside the penetrating fenestrae and the soft cell body itself. However, this mode is not workable for fenestra imaging of LSECs on soft substrates, since the stiffness difference between dish bottom and cell body disappeared in this case (data not shown). Meanwhile, the two imaging methods of contact mode and PF-QNM mode cost similar time at same resolution and have their own advantages for fenestra visualizing (Table S2). The former favors the fenestra identification on soft substrate while the latter facilitates the visualization of the dynamic fenestra morphology in live LSECs. Since the fenestra imaging on stiffness-varied substrates is the focus of this work, only the LSEC morphology images collected from contact mode were utilized in subsequent deep-learning studies.

Moreover, to apply this AFM imaging assay of LSEC fenestrae in various biological issues, acquiring high-resolution images in a confined cell region should be balanced with collecting global information over the entire cell as possible. The high-resolution images (Fig. 3B) provides clear visualization of fenestrae and favors the fenestra recognition upon high contrast between fenestrae and the surrounding membrane. By contrast, the large-area imaging in LESC morphology (Fig. 4A, C) displays global distribution of LSEC fenestrae and facilitates the understanding of regulatory mechanisms. Thus, those intermediate-magnification images of LSEC morphology on various substrates were used for developing the fenestra recognition program upon deep-learning below.

\subsection{FCN-aided fenestra recognition}

Even with the optimized AFM imaging assay as above, it is still labor-consuming to summary the statistics of LSES fenestra distribution via manual recognition since several-hundred fenestrae usually exist in a single LSEC. We thus developed a FCN-aided fenestra recognition program upon deep-learning to conduct automatic recognition. Evidently, the FCN model was converged quickly as the number of epoches was increased beyond 15 (Fig. S6). The FCN model after twice training with testing set images was able to recognize accurately the majority of fenestrae in the localized images of LSEC morphology (Fig. 5). This trained model was further validated using validating set images, which returned the enhanced four parameters of FCN criteria 
(Table 1). That is, the values of pixel acc., mean acc., mean $I U$, and F.W.IU were raised to $0.980,0.688,0.658$, and 0.963 , respectively, in this validated FCN2 model.

Specifically, applying the FCN1 was able to recognize 914 objects in the testing set of 996 fenestrae manually labeled, of which 750 objects matched those manual labels to yield a Dice of 0.668 and a $F_{1}$ score of 0.786 (Table 1). Those non-fenestra objects so recognized were then eliminated by optimizing the object area threshold via setting the threshold gradients. Results indicated that the Dice and $F_{1}$ score reached highest values at the threshold of 0.2 and 0.3 and the effective fenestra information was retained at the threshold of 0.2 (data not shown). Setting the optimized threshold of 0.2 returned 713 recognized objects, of which 641 objects matched the manual labels to yield the enhanced Dice of 0.727 (Table 1). After the second-run training, the number of objects recognized by FCN2 and the matched fenestra number increased to 967 and 813, respectively, resulting in the higher Dice (0.773) and $\mathrm{F}_{1}$ score (0.831) than FCN1. As a result, the median and quartile of Dice and $F_{1}$ score were higher in FCN2 than FCN1 with concentrated distributions, and $F_{1}$ score was slightly higher at the threshold of 0.2 than those at 0 and 0.3 (Fig. 6A, B). This program enabled us to recognize the majority of fenestrae from AFM images after second training at an accuracy of $81.6 \%$ ( $=\#$ of $\mathrm{FCN}$ recognition at 0.2 area threshold / \# of manual labels).

More biologically, the estimated porosity, number of fenestrae, and fenestra diameter distribution were compared between manual annotation and FCN recognition given by the diameter of those fenestrae $<500 \mathrm{~nm}$. Similar fluctuating patterns of the three parameters were observed between the two methods, and the data from FCN2 model were most likely overlapped with those data from label group. Here lower porosity and number and higher peak fenestra diameter were presented after first FCN training but the three values quickly converged to those in label group after second training, consistent with those in the literatures (i.e., $140 \mathrm{~nm}$ of peak fenestra diameter) $[8,18]$. In fenestra diameter distributions, numerous fenestrae less than $60 \mathrm{~nm}$ were first presented at the threshold of 0 but removed at threshold of 0.2 or 0.3 , resulting in similar lower limit of $60 \mathrm{~nm}$ of fenestra diameter in label group. This analysis further confirmed the necessity of setting a reasonable area threshold. Additional advantage for FCN model was to save time on recognizing the fenestrae. As an example, the average time of manual recognition in a single localized image of LSECs was $15 \mathrm{~min}$, which spent $210 \mathrm{~min}$ for total 14 images. By contrast, it took $8.6 \mathrm{~min}$ to complete the entire recognition using FCN2 model.

Collectively, the FCN models demonstrated, for the first time, automatic recognition and quantitation of fenestrae in localized images of LSEC morphology. Technically, the current segmentations performed well on tracing fenestrae and reached the average levels of existing FCN models (Tables S3, 4, 5) [21,34,39,40]. As shown in Fig. 5, the number, shape and size of fenestrae were comparable to those in the original images and label group after second FCN training, further supporting their performances. In addition, the neighboring fenestrae can be segmented as independent individuals but not a merged ensemble (green arrows in Fig. 5). Moreover, these FCN-aided readouts can be employed directly to estimate the biologically-relevant porosity, number of fenestrae, and diameter frequency, since they were quite similar to those in label group with a majority of overlapping between the two data sets (Fig. 6C-E).

\section{Conclusions}

Optimized AFM imaging was able to visualize accurately the fenestrated morphology of LSECs. Large-area acquisition of morphology images $(16.6 \times 16.6 \mu \mathrm{m})$ displayed global fenestra information over an entire cell especially on soft substrate. A fenestra recognition program upon FCN training was developed to recognize fenestrae at comparable accuracy with manual annotation on reduced time. FCN-aided automatic recognition favored the statistical calculations of the porosity, number of fenestrae and distribution of fenestra diameter.

\section{Funding}

This work was supported by the National Natural Science Foundation of China [grant numbers 91642203, 31627804, 31661143044, 31870930]; the Chinese Academy of Sciences [grant numbers QYZDJ-SSW-JSC018 and XDB22040101]; and the National Key Research and Development Program of China [grant number 2017YFC0108500].

\section{Declaration of Competing Interest}

The authors declare no competing financial interests.

\section{Appendix A. Supplementary data}

Supplementary data to this article can be found online at https:// doi.org/10.1016/j.bbagen.2020.129702.

\section{References}

[1] D. Svistounov, A. Warren, G.P. McNerney, D.M. Owen, D. Zencak, S.N. Zykova, H. Crane, T. Huser, R.J. Quinn, B. Smedsrod, D.G. Le Couteur, V.C. Cogger, The relationship between fenestrations, sieve plates and rafts in liver sinusoidal endothelial cells, PLoS One 7 (2012) e46134, , https://doi.org/10.1371/journal.pone. 0046134.

[2] S. Shetty, P.F. Lalor, D.H. Adams, Liver sinusoidal endothelial cells - gatekeepers of hepatic immunity, Nat. Rev. Gastroenterol. Hepatol. 15 (2018) 555-567, https:// doi.org/10.1038/s41575-018-0020-y.

[3] J. Poisson, S. Lemoinne, C. Boulanger, F. Durand, R. Moreau, D. Valla, P.E. Rautou, Liver sinusoidal endothelial cells: physiology and role in liver diseases, J. Hepatol. 66 (2017) 212-227, https://doi.org/10.1016/j.jhep.2016.07.009.

[4] S.N. Hilmer, V.C. Cogger, R. Fraser, A.J. Mclean, D. Sullivan, D.G. Le Couteur, Age related changes in the hepatic sinusoidal endothelium impede lipoprotein transfer in the rat, Hepatology 42 (2005) 1349-1354, https://doi.org/10.1002/hep.20937.

[5] V.C. Cogger, S.N. Hilmer, D. Sullivan, M. Muller, R. Fraser, D.G. Le Couteur, Hyperlipidemia and surfactants: the liver sieve is a link, Atherosclerosis 189 (2006) 273-281, https://doi.org/10.1016/j.atherosclerosis.2005.12.025.

[6] B. Zapotoczny, K. Szafranska, K. Owczarczyk, E. Kus, S. Chlopicki, M. Szymonski, Atomic force microscopy reveals the dynamic morphology of fenestrations in live liver sinusoidal endothelial cells, Sci. Rep. 7 (2017) 7994, https://doi.org/10.1038/ s41598-017-08555-0.

[7] J.R. Hu, S.Q. Lü, S.L. Feng, M. Long, Flow dynamics analyses of pathophysiological liver lobules using porous media theory, Acta Mech. Sinica 33 (2017) 823-832, https://doi.org/10.1007/s10409-017-0674-7.

[8] B. Zapotoczny, K. Owczarczyk, K. Szafranska, E. Kus, S. Chlopicki, M. Szymonski, Morphology and force probing of primary murine liver sinusoidal endothelial cells, J. Mol. Recognit. 30 (2017) e2610, , https://doi.org/10.1002/jmr.2610.

[9] C.I. Oiea, V. Monkemollera, W. Hubner, M. Schuttpelz, H. Mao, B.S. Ahluwalia, T.R. Huser, P. McCourt, New ways of looking at very small holes - using optical nanoscopy to visualize liver sinusoidal endothelial cell fenestrations, Nanophotonics 7 (2018) 575-596, https://doi.org/10.1515/nanoph-2017-0055.

[10] H. Yokomori, New insights into the dynamics of sinusoidal endothelial fenestrae in liver sinusoidal endothelial cells, Med. Mol. Morphol. 41 (2008) 1-4, https://doi. org/10.1007/s00795-007-0390-7.

[11] E. Wisse, R.B. De Zanger, R. Jacobs, R.S. McCuskey, Scanning electron microscope observations on the structure of portal veins, sinusoids and central veins in rat liver, Scan. Electron Microsc. 3 (1983) 1441-1452.

[12] J.Y. Fu, C.M. Zhang, Super-resolution microscopy: successful applications in centrosome study and beyond, Biophys. Rep. 5 (2019) 235-243, https://doi.org/10. 1007/s41048-019-00101-x.

[13] V. Monkemoller, M. Schuttpelz, P. McCourt, K. Sorensen, B. Smedsrod, T. Huser, Imaging fenestrations in liver sinusoidal endothelial cells by optical localization microscopy, Phys. Chem. Chem. Phys. 16 (2014) 12576-12581, https://doi.org/10. 1039/c4cp01574f.

[14] V. Monkemoller, C. Oie, W. Hubner, T. Huser, P. McCourt, Multimodal super-resolution optical microscopy visualizes the close connection between membrane and the cytoskeleton in liver sinusoidal endothelial cell fenestrations, Sci. Rep. 5 (2015) 16279, https://doi.org/10.1038/srep16279.

[15] J. Di Martino, P. Mascalchi, P. Legros, S. Lacomme, E. Gontier, P. Bioulac-Sage, C. Balabaud, V. Moreau, F. Saltel, STED microscopy: a simplified method for liver sinusoidal endothelial fenestrae analysis, Biol. Cell. 110 (2018) 159-168, https:// doi.org/10.1111/boc.201800016.

[16] Z.F. You, L. Zhou, W.J. Li, C.Y. Huang, Y.N. Du, Mechanical microenvironment as a key cellular regulator in the liver, Acta Mech. Sinica 35 (2019) 289-298, https:// doi.org/10.1007/s10409-019-00857-y.

[17] A.J. Ford, G. Jain, P. Rajagopalan, Designing a fibrotic microenvironment to investigate changes in human liver sinusoidal endothelial cell function, Acta 
Biomater. 24 (2015) 220-227, https://doi.org/10.1016/j.actbio.2015.06.028.

[18] B. Zapotocznya, K. Szafranskaa, E. Kusb, S. Chlopickib, M. Szymonskia, Quantification of fenestrations in liver sinusoidal endothelial cells by atomic force microscopy, Micron 101 (2017) 48-53, https://doi.org/10.1016/j.micron.2017.06. 005.

[19] B. Zapotoczny, K. Szafranska, E. Kus, F. Braet, E. Wisse, S. Chlopicki, M. Szymonski, Tracking fenestrae dynamics in live murine liver sinusoidal endothelial cells, Hepatology 69 (2019) 876-888, https://doi.org/10.1002/hep.30232.

[20] T. Falk, D. Mai, R. Bensch, O. Cicek, A. Abdulkadir, Y. Marrakchi, A. Böhm, J. Deubner, Z. Jäckel, K. Seiwald, A. Dovzhenko, O. Tietz, C. Dal Bosco, S. Walsh, D. Saltukoglu, T.L. Tay, M. Prinz, K. Palme, M. Simons, I. Diester, T. Brox, O. Ronneberger, U-net: deep learning for cell counting, detection, and morphometry, Nat. Methods 16 (2019) 67-70, https://doi.org/10.1038/s41592-0190356-4.

[21] E. Shelhamer, J. Long, T. Darrell, Fully convolutional networks for semantic segmentation, IEEE T. Pattern Anal. 39 (2017) 640-651, https://doi.org/10.1109/ TPAMI.2016.2572683.

[22] W.D. Xie, J.A. Noble, A. Zisserman, Microscopy cell counting and detection with fully convolutional regression networks, Comput. Method. Biomec. 6 (2016) 283-292, https://doi.org/10.1080/21681163.2016.1149104.

[23] Y. Du, N. Li, H. Yang, C.H. Luo, Y.X. Gong, C.F. Tong, Y.X. Gao, S.Q. Lü, M. Long, Mimicking liver sinusoidal structures and functions using a 3D-configured microfluidic chip, Lab Chip 17 (2017) 782-794, https://doi.org/10.1039/c6lc01374k.

[24] C.F. Tong, Y. Zhang, S.Q. Lü, N. Li, Y.X. Gong, H. Yang, S.L. Feng, Y. Du, D.D. Huang, M. Long, Binding of intercellular adhesion molecule 1 to beta(2)-integrin regulates distinct cell adhesion processes on hepatic and cerebral endothelium, Am. J. Phys. Cell Phys. 315 (2018) C409-C421, https://doi.org/10. 1152/ajpcell.00083.2017.

[25] N.D. Evans, C. Minelli, E. Gentleman, V. LaPointe, S.N. Patankar, M. Kallivretaki, X. Chen, C.J. Roberts, M.M. Stevens, Substrate stiffness affects early differentiation events in embryonic stem cells, Eur. Cells Mater. 18 (2009) 1-13, https://doi.org/ 10.22203/ecm.v018a01.

[26] L. Przybylaa, J.N. Lakins, R. Sunyerb, X. Trepat, V.M. Weaver, Monitoring developmental force distributions in reconstituted embryonic epithelia, Methods 94 (2016) 101-113, https://doi.org/10.1016/j.ymeth.2015.09.003.

[27] Y.H. Xu, D.D. Huang, S.Q. Lü, Y. Zhang, M. Long, Mechanical features of endothelium regulate cell adhesive molecule-induced calcium response in neutrophils, APL Bioeng. 3 (2019) 016104, , https://doi.org/10.1063/1.5045115.

[28] S.S. Desai, J.C. Tung, V.X. Zhou, J.P. Grenert, Y. Malato, M. Rezvani, R. EspanolSuner, H. Willenbring, V.M. Weaver, T.T. Chang, Physiological ranges of matrix rigidity modulate primary mouse hepatocyte function in part through hepatocyte nuclear factor 4 alpha, Hepatology 64 (2016) 261-275, https://doi.org/10.1002/ hep. 28450.

[29] Z. Li, Y.W. Gong, S.J. Sun, Y. Du, X.F. Liu, M. Long, Differential regulation of stiffness, topography, and dimension of substrates in rat mesenchymal stem cells, Biomaterials 34 (2013) 7616-7625, https://doi.org/10.1016/j.biomaterials.2013. 06.059.

[30] J.N. Lakins, A.R. Chin, V.M. Weaver, Exploring the link between human embryonic stem cell organization and fate using tension-calibrated extracellular matrix functionalized polyacrylamide gels, Methods Mol. Biol. 916 (2012) 317-350, https:// doi.org/10.1007/978-1-61779-980-8_24.

[31] J.R. Hu, D.D. Huang, Y. Zhang, S.Q. Lü, M. Long, Global mapping of live cell mechanical features using PeakForce QNM AFM, Biophys. Rep. (2020), https://doi. org/10.1007/s41048-019-00103-9.

[32] Y.Q. Jia, E. Shelhamer, J. Donahue, S. Karayev, J. Long, R. Girshick, S. Guadarrama, T. Darrell, Caffe: convolutional architecture for fast feature embedding, New York: Assoc Comp. Mach. (2014) 675-678, https://doi.org/10.1145/2647868.2654889.

[33] K.H. Zou, S.K. Warfield, A. Bharatha, C.M.C. Tempany, M.R. Kaus, S.J. Haker, W.M. Wells, F.A. Jolesz, R. Kikinis, Statistical validation of image segmentation quality based on a spatial overlap index - scientific reports, Acad. Radiol. 11 (2004) 178-189, https://doi.org/10.1016/S1076-6332(03)00671-8.

[34] X. Yang, H. Li, Y. Yu, X. Luo, T. Huang, X. Yang, Automatic pixel-level crack detection and measurement using fully convolutional network, Comput-Aided Civ. Inf. 33 (2018) 1090-1109, https://doi.org/10.1111/mice.12433.

[35] E. Kus, P. Kaczara, I. Czyzynska-Cichon, K. Szafranska, B. Zapotoczny, A. Kij, A. Sowinska, J. Kotlinowski, L. Mateuszuk, E. Czarnowska, M. Szymonski, S. Chlopicki, LSEC fenestrae are preserved despite pro-inflammatory phenotype of liver sinusoidal endothelial cells in mice on high fat diet, Front. Physiol. 10 (2019) 6, https://doi.org/10.3389/fphys.2019.00006.

[36] J.W. Wang, D.Y. Lü, D.B. Mao, M. Long, Mechanomics: an emerging field between biology and biomechanics, Protein Cell 5 (2014) 518-531, https://doi.org/10. 1007/s13238-014-0057-9.

[37] F. Braet, D.J. Taatjes, E. Wisse, Probing the unseen structure and function of liver cells through atomic force microscopy, Semin. Cell Dev. Biol. 73 (2018) 13-30, https://doi.org/10.1016/j.semcdb.2017.07.001.

[38] J.R. Hu, S.B. Chen, W.H. Hu, S.Q. Lü, M. Long, Mechanical point loading induces cortex stiffening and actin reorganization, Biophys. J. 117 (2019) 1-14, https://doi org/10.1016/j.bpj.2019.09.012.

[39] R. Karthik, U. Gupta, A. Jha, R. Rajalakshmi, R. Menaka, A deep supervised approach for ischemic lesion segmentation from multimodal MRI using fully convolutional network, Appl. Soft Comput. 84 (2019) 105685, https://doi.org/10. 1016/j.asoc.2019.105685.

[40] M. Signaevsky, M. Prastawa, K. Farrell, N. Tabish, E. Baldwin, N. Han, M.A. Iida, J. Koll, C. Bryce, D. Purohit, V. Haroutunian, A.C. McKee, T.D. Stein, C.L. White, J. Walker, T.E. Richardson, R. Hanson, M.J. Donovan, C. Cordon-Cardo, J. Zeineh, G. Fernandez, J.F. Crary, Artificial intelligence in neuropathology: deep learningbased assessment of tauopathy, Lab. Investig. 99 (2019) 1019-1029, https://doi. org/10.1038/s41374-019-0202-4. 